\title{
Viabilidade econômica do uso da glicerina bruta em dietas para cordeiros terminados em confinamento
}

\section{Economic viability of crude glycerin in diets for lambs finished in feedlot}

\author{
Mauriceia Costa Carvalho Barros ${ }^{1 *}$; Jair de Araújo Marques ${ }^{2}$; \\ Robério Rodrigues Silva ${ }^{3}$; Fabiano Ferreira da Silva ${ }^{3}$; Lucas Teixeira Costa ${ }^{1}$; \\ Gilmara Santos Guimarães ${ }^{4}$; Luciano Lemos da Silva ${ }^{4}$; \\ José Joaquim Nunes Gusmão ${ }^{5}$
}

\begin{abstract}
Resumo
O objetivo foi avaliar a viabilidade econômica de níveis crescentes de glicerina bruta (GB) em dietas para cordeiros na fase de terminação em confinamento. O experimento foi desenvolvido na Universidade Estadual do Sudoeste da Bahia, Itapetinga-BA. Foram utilizados 25 cordeiros mestiços Santa Inês x Dorper, machos castrados, com $24 \pm 2,0 \mathrm{~kg}$, alojados em baias individuais. O delineamento utilizado foi inteiramente casualizado, com cinco tratamentos e cinco repetições. Os tratamentos consistiram de níveis crescentes da GB na dieta $(0 ; 2,65 ; 5,33 ; 8,06$ e $10,84 \%)$ e o volumoso utilizado foi o feno de Tifton 85. As dietas foram calculadas para atender às exigências nutricionais, visando um ganho de 200 $\mathrm{g} /$ dia. A análise foi realizada a partir da utilização dos indicadores econômicos como o Valor Presente Líquido (VPL) e Taxa Interna de Retorno (TIR). O consumo de matéria seca dos animais diminuiu linearmente $(\mathrm{P}<0,05)$ com o aumento dos níveis de glicerina na dieta, porém, não foi observada influência $(\mathrm{P}>0,05)$ para o desempenho e, consequentemente, para a produção de carne. Nenhum dos tratamentos apresentou viabilidade econômica.
\end{abstract}

Palavras-chave: Custos, produtividade, sistema de produção, biodiesel

\footnotetext{
Abstract

The aim was to evaluate the economic viability of increasing levels of crude glycerin $(\mathrm{CG})$ in diets for finishing lambs. The trial was carried out at Southwest State University of Bahia, Itapetinga-BA. Twenty five crossbred, Santa Inês x Dorper, with $24 \pm 2,0 \mathrm{~kg}$, were housed in individual pens. The experimental design was completely randomized with five treatments and five replications. Treatments consisted of increasing levels of dietary CG $(0,2.65,5.33,8.06$ and $10.84 \%)$ and the roughage used was the Tifton 85 hay. Diets were formulated to meet the nutritional requeriments, aiming a gain of $200 \mathrm{~g} \mathrm{day}^{-1}$. The analysis was based on use of economic indicators Net Present Value (NPV) and Internal Rate of Return

${ }^{1}$ Discentes do Curso de Doutorado do Programa de Pós-Graduação em Zootecnia, Universidade Estadual do Sudoeste da Bahia, UESB, Campus Itapetinga, BA. E-mail: ceacarvalho@ig.com.br; 1tcosta80@yahoo.com.br

${ }^{2}$ Prof. Adjunto, Dept ${ }^{\mathrm{o}}$ de Zootecnia, Universidade Federal do Recôncavo da Bahia, UFRB, Campus Cruz das Almas, BA. E-mail: jdmarques@hotmail.com

${ }^{3}$ Profs. Titulares, Dept ${ }^{\circ}$ de Zootecnia, UESB, Campus Itapetinga, BA. E-mail: rrsilva.uesb@hotmail.com; ffsilvauesb@hotmail. com

${ }^{4}$ Discentes do Curso de Mestrado do Programa de Pós-Graduação em Zootecnia, UESB, Campus Itapetinga, BA. E-mail: gguimaraes114@hotmail.com; lucianolemos-zootec@hotmail.com

${ }^{5}$ Discente do Curso de Graduação em Zootecnia, UESB, Campus Itapetinga, BA. E-mail: jjngusmao@zootecnista.com.br

* Autor para correspondência
} 
(IRR). The animals dry matter intake decreased linearly $(\mathrm{P}<0.05)$ as the glycerin levels increased in the diet. However, there was no effect $(\mathrm{P}>0.05)$ on performance and meat production results. None of the treatments showed economic viability.

Key words: Costs, productive, systems of production, biodiesel

\section{Introdução}

O Nordeste brasileiro destaca-se por ser uma região com grande aptidão para a exploração de ovinos, sendo esta atividade considerada como primordial no suprimento alimentar e na geração de renda. No entanto, o baixo potencial genético dos rebanhos, associado à escassez de pastos na estação seca e as práticas de manejo inadequadas concorrem para os baixos índices de produtividade e de rentabilidade.

O confinamento vem sendo considerado como uma alternativa viável para esse tipo de exploração, por permitir a intensificação da produção animal associada à redução do tempo para produzir carcaça com qualidade exigida pelo mercado consumidor e, consequentemente, acelerar o retorno do capital aplicado. No entanto, tem-se a elevação no custo de produção dos animais criados neste sistema, o que possivelmente pode ser considerado um entrave para a produção animal, sendo necessária a busca por alternativas alimentares que viabilizem a utilização do confinamento.

A glicerina bruta é um coproduto da produção de biodiesel com baixo custo e tem surgido como opção para utilização na dieta de cordeiros em terminação, em substituição a concentrados energéticos (KERR et al., 2007). A utilização de coprodutos provenientes da produção de biocombustíveis é uma opção importante para reduzir o impacto ambiental, além de possibilitar reduzir o custo de produção de proteína de alto valor biológico, como é o caso da carne ovina.

O desempenho dos animais e as características da carcaça são frequentemente estudados em sistemas de terminação de cordeiros. Entretanto, a análise econômica não é realizada com a mesma frequência, apesar de ser importante e permitir o conhecimento detalhado dos custos de produção, auxiliando na melhoria da lucratividade da atividade.

Segundo Peres et al. (2004), o valor presente líquido (VPL) e a taxa interna de retorno (TIR) são indicadores econômicos que podem ser utilizados para a avaliação financeira de sistemas de produção. O VPL é isento de falhas técnicas e, por isso, é considerado um critério rigoroso de avaliação de projetos (NORONHA, 1987; CONTADOR, 1988). O VPL corresponde à soma algébrica dos valores do fluxo de caixa de um projeto, atualizados à taxa ou às taxas de desconto do período em questão. Segundo esse indicador, um projeto é viável se apresentar um VPL positivo, sendo mais atrativo aquele projeto que apresentar o maior VPL positivo. Contador (1988) definiu a taxa interna de retorno (TIR) como a taxa de juros que iguala a zero o VPL de um projeto, ou seja, é a taxa de desconto que iguala o valor presente dos benefícios de um projeto ao valor presente de seus custos. Um projeto é viável e deve ser adotado quando sua TIR é igual ou maior que o custo de oportunidade dos recursos para sua implantação.

No presente estudo, objetivou-se avaliar as respostas econômicas dos níveis crescentes de glicerina bruta na dieta de cordeiros na fase de terminação em confinamento.

\section{Material e Métodos}

O experimento foi desenvolvido no setor de ovinocultura da Universidade Estadual do Sudoeste da Bahia - UESB, campus de Itapetinga-BA, durante o período de junho a setembro de 2010. 
Foram utilizados 25 cordeiros mestiços Santa Inês x Dorper, com seis meses de idade, machos, castrados, com peso corporal de $24 \pm 2,0 \mathrm{~kg}$ e alojados em baias individuais. O delineamento utilizado foi inteiramente casualizado, com cinco tratamentos e cinco repetições. Os tratamentos consistiram em diferentes níveis de glicerina bruta na dieta $(0 ; 2,65 ; 5,33 ; 8,06$ e $10,84 \%)$, com base na matéria seca.

As dietas foram fornecidas duas vezes ao dia, às 07:00 e às 16:00 horas, na forma de ração completa. O período experimental foi de 100 dias, sendo os primeiros 16 dias para adaptação dos animais às instalações, às dietas experimentais e ao manejo, e os 84 dias restantes destinados à avaliação e coleta de dados, divididos em três períodos de 28 dias.

Neste período, foram feitos os ajustes de consumo por meio de pesagem do alimento fornecido e das sobras, permitindo ingestão ad libitum, admitindo sobras de, no máximo, $10 \%$ da quantidade ofertada. A água esteve, permanentemente, à disposição dos animais, fornecida em baldes plásticos.

As dietas foram formuladas de acordo com as recomendações nutricionais do NRC (2007), visando um ganho médio diário de $0,2 \mathrm{~kg}$. Foi utilizado o feno de capim Tifton 85 como volumoso e os concentrados foram compostos por milho, farelo de soja, uréia, mistura mineral e glicerina (Tabelas 1 e 2).

Tabela 1. Composição percentual dos ingredientes e composição química das dietas.

\begin{tabular}{cccccc}
\hline & \multicolumn{5}{c}{ Níveis de glicerina (\%MS da dieta) } \\
\cline { 2 - 5 } Alimentos & 0 & 2,65 & 5,33 & 8,06 & 10,84 \\
\hline Feno de Tifton & 51,78 & 51,76 & 51,75 & 51,74 & 51,73 \\
Farelo de milho & 45,15 & 41,91 & 38,61 & 35,27 & 31,88 \\
Glicerina bruta & 0 & 2,65 & 5,33 & 8,06 & 10,84 \\
Farelo de soja & 2,02 & 2,60 & 3,19 & 3,80 & 4,41 \\
Sal mineral $^{1}$ & 0,34 & 0,34 & 0,35 & 0,35 & 0,35 \\
Ureia $_{\text {Calcário }}$ & 0,35 & 0,35 & 0,35 & 0,35 & 0,36 \\
Fosfato bicálcio $_{\text {Nutrientes }}^{2}$ & 0,22 & 0,20 & 0,18 & 0,16 & 0,13 \\
MS & 0,16 & 0,19 & 0,23 & 0,27 & 0,30 \\
PB & \multicolumn{5}{c}{ Composição química (\%) } \\
EE & 90,38 & 90,45 & 90,57 & 90,63 & 90,67 \\
FDNcp & 11,90 & 12,01 & 12,07 & 12,15 & 12,21 \\
MO & 2,80 & 3,26 & 4,58 & 5,39 & 6,79 \\
CNFcp & 47,50 & 47,53 & 47,20 & 47,29 & 46,94 \\
MM & 94,81 & 94,69 & 94,60 & 94,54 & 94,39 \\
\hline & 32,60 & 31,89 & 30,75 & 29,71 & 28,45 \\
\hline
\end{tabular}

${ }^{1}$ Níveis de garantia (nutrientes $/ \mathrm{kg}$ ): cálcio-150g; enxofre-12g; fósforo-65g; magnésio-6.000mg; sódio- 107g; cobre- 100mg; cobalto-175mg; ferro-1000mg; flúor máximo-650mg; iodo-175mg; manganês-1440mg; selênio-27mg e zinco- $6000 \mathrm{mg}$. ${ }^{2} \mathrm{MS}=$ matéria seca, $\mathrm{PB}=$ proteína bruta, $\mathrm{FDNcp}=$ fibra em detergente neutro isenta de cinzas e proteína, $\mathrm{MO}=$ matéria orgânica, $\mathrm{CNF} c \mathrm{p}$ = carboidratos não fibrosos isentos de cinza e proteína e $\mathrm{MM}=$ matéria mineral.

Fonte: Elaboração dos autores. 
As informações necessárias para a elaboração deste trabalho e composição dos custos, bem como os dados utilizados (preços, vida útil etc) foram coletados junto aos produtores rurais, técnicos de extensão rural e estabelecimentos comerciais da região, de janeiro a dezembro de 2010 (Tabela 3, Tabela 4 e Tabela 5, respectivamente).
Tabela 2. Composição físico-química da glicerina bruta utilizada para compor os concentrados da dieta.

\begin{tabular}{lc}
\hline Item $(\% \mathrm{MN})^{*}$ & Teor $(\%)$ \\
\hline Glicerol & 43,9 \\
Metanol & 6,0 \\
Ácidos graxos totais & 33,6 \\
Água & 9,0 \\
Proteína bruta & 0,2 \\
Matéria mineral & 7,3 \\
Densidade $\mathrm{g} \mathrm{cm}^{-1}$ & 0,95 \\
\hline
\end{tabular}

*Matéria Natural

Fonte: Elaboração dos autores.

Tabela 3. Preços de insumos e serviços utilizados no experimento.

\begin{tabular}{lcc}
\hline \multicolumn{1}{c}{ Descrição } & Unidade & Valor unitário (R\$) \\
\hline Mão-de-obra & $\mathrm{d} / \mathrm{H}^{1}$ & 30,00 \\
Feno & $\mathrm{Kg} \mathrm{de} \mathrm{MS}$ & 0,36 \\
Energia & $\mathrm{kW} / \mathrm{h}^{2}$ & 0,27 \\
Concentrado com 0\% glicerina & $\mathrm{Kg} \mathrm{de} \mathrm{MS}$ & 0,48 \\
Concentrado com 2,65\% glicerina & $\mathrm{Kg}$ de $\mathrm{MS}$ & 0,47 \\
Concentrado com 5,33\% glicerina & $\mathrm{Kg} \mathrm{de} \mathrm{MS}$ & 0,46 \\
Concentrado com 8,06\% glicerina & $\mathrm{Kg}$ de MS & 0,45 \\
Concentrado com 10,84\% glicerina & $\mathrm{Kg}$ de MS & 0,44 \\
\hline
\end{tabular}

${ }^{1} \mathrm{~d} / \mathrm{H}=$ dia por homem; ${ }^{2} \mathrm{kw} / \mathrm{h}=$ quilowatts por hora

Fonte: Elaboração dos autores.

Tabela 4. Preço dos ingredientes, por quilo de matéria seca, dos concentrados utilizados no experimento.

\begin{tabular}{lc}
\hline Descrição & Preço unitário (R\$) \\
\hline Farelo de milho & 0,45 \\
Farelo de soja & 0,75 \\
Uréia & 2,20 \\
Sal comum & 0,42 \\
Calcário calcítico & 0,22 \\
Fosfato bicálcio & 2,60 \\
Glicerina bruta & 0,20 \\
\hline
\end{tabular}

Fonte: Elaboração dos autores.
Foram consideradas, para avaliação do custo de produção, as metodologias de custos operacionais utilizada pelo IPEA (MATSUNAGA et al., 1976). A depreciação de benfeitorias, máquinas e equipamentos foram estimadas pelo método linear de cotas fixas, com valor final igual a zero. Para a remuneração do capital, utilizou-se a taxa de juros real de $6 \%$ ao ano. 
Tabela 5. Vida útil e valor de benfeitorias, máquinas e equipamentos, quantidades utilizadas no experimento e seus valores.

\begin{tabular}{|c|c|c|c|c|}
\hline Discriminação & $\begin{array}{l}\text { Vida útil } \\
\text { (dias) }\end{array}$ & $\begin{array}{l}\text { Valor unitário } \\
(\mathrm{R} \$)\end{array}$ & $\begin{array}{c}\text { Quantidade } \\
\text { utilizada (unid.) }\end{array}$ & Valor total $(\mathrm{R} \$)$ \\
\hline Balança para pesagem dos ovinos & 5475 & 800,00 & 1 & 800,00 \\
\hline Triturador forrageiro & 5475 & $3.900,00$ & 1 & $3.900,00$ \\
\hline Garfo de quatro dentes & 730 & 12,00 & 1 & 12,00 \\
\hline Pá & 730 & 6,10 & 1 & 6,10 \\
\hline Carrinho de mão & 730 & 75,00 & 1 & 75,00 \\
\hline Ovinos & - & 90,00 & 25 & $2.250,00$ \\
\hline Utilidade de pequeno valor & 730 & 35,30 & 1 & 35,30 \\
\hline Galpão de confinamento de $2500 \mathrm{~m}^{2}$ & 5475 & $5.000,00$ & 1 & $5.000,00$ \\
\hline Capital fixo investido & & & & $12.078,40$ \\
\hline
\end{tabular}

Fonte: Elaboração dos autores.

Utilizaram-se, para efeito de estudo da análise econômica, dois indicadores econômicos: o VPL (valor presente líquido) e a TIR (taxa interna de retorno). O VPL foi calculado segundo a equação:

$$
V P L=\sum_{t=0}^{n} \frac{V F L}{(1+r) t}
$$

Em que: $\mathrm{VPL}=$ valor presente líquido; $\mathrm{VFL}=$ valor do fluxo líquido (diferença entre entradas e saídas); $\mathrm{n}=$ número de fluxos; $\mathrm{r}=$ taxa de desconto; $\mathrm{t}=$ período de análise $(\mathrm{i}=1,2,3 \ldots)$.

No cálculo do VPL, aplicaram-se três taxas de desconto sobre o fluxo líquido mensal de cada sistema de produção. As taxas adotadas foram 6,10 e $12 \%$ ao ano.

Para a TIR, segundo os critérios de aceitação, quanto maior for o resultado obtido no projeto, maior será a atratividade para sua implantação. Assim, a TIR é o valor de r que iguala a zero a expressão:

$$
V P L=V F_{0}+\frac{V F_{1}}{(1+r)^{1}}+\frac{V F_{2}}{(1+r)^{2}}+\frac{V F_{3}}{(1+r)^{3}}+\ldots+\frac{V F_{n}}{(1+r)^{n}}
$$

Em que: $\mathrm{VF}=$ fluxos de caixa líquido $(0,1,2$, $3, \ldots, n) ; r=$ taxa de desconto.

Para cálculo da TIR e do VPL, fez-se uma simulação de um ano para estudo de características econômicas, sendo computada, assim, a depreciação de benfeitorias e máquinas neste período.

Para realizar os cálculos de fluxo de caixa, foi admitido o valor de dez reais $(\mathrm{R} \$ 10,00)$ por unidade de peso $(\mathrm{kg})$ da carcaça ovina, sendo este valor praticado no mercado de Itapetinga - BA, durante o período em que foi realizado o abate dos animais.
As análises estatísticas foram realizadas utilizando-se o programa SAEG (2007) por meio da análise de variância e regressão, adotando-se o nível de 5\% de significância para o tratamento. Os critérios utilizados para escolha dos modelos de regressão consideraram inicialmente a significância para o tratamento, posteriormente consideraram a adequação do modelo aos fenômenos estudados, os valores dos coeficientes de determinação ajustados e a significância dos parâmetros da regressão pelo teste t. 


\section{Resultados e Discussão}

$\mathrm{O}$ aumento de inclusão de glicerina bruta na dieta apresentou efeito linear decrescente $(\mathrm{P}<0,05)$ sobre o consumo de matéria seca (CMS) (Tabela 6). Este resultado foi semelhante ao encontrado por Lage et al. (2010), que avaliaram níveis de glicerina semelhantes ao do presente trabalho (zero, 3,0;6,0;9,0 e $12 \%$ ) e por Gunn et al. (2010), que trabalharam com níveis de inclusão da glicerina $(0,15,30$ e $45 \%)$ em dietas de ovinos em confinamento.

Pyatt, Doane e Cecava (2007) constataram uma redução no CMS, quando dietas contendo $10 \%$ de glicerina bruta foram utilizadas na fase de terminação de bovinos em confinamento. Da mesma forma, Parsons, Shelor e Drouillard (2009) relataram uma diminuição linear na ingestão de MS, quando a glicerina bruta foi incluída em quantidade superior a $2 \%$ de MS na dieta de novilhas em acabamento.

No presente trabalho, o consumo de matéria seca variou de $0,580 \mathrm{~kg} \mathrm{dia}^{-1}$ (dieta com 10,84\% de glicerina) a $0,943 \mathrm{~kg} /$ dia (dieta controle), essa variação ocorreu, possivelmente, pelo fato da glicerina utilizada no presente estudo ter sido de baixa pureza, acreditando-se que os níveis de glicerol, metanol e extrato etéreo na dieta contribuíram de forma isolada ou em conjunto para influenciarem o CMS pelos animais.

Com relação ao ganho médio diário (GMD) e conversão alimentar ( $\mathrm{kg} \mathrm{MS} \mathrm{kg}{ }^{-1}$ carne), não foi verificado efeito $(\mathrm{P}>0,05)$ dos níveis de inclusão de glicerina, obtendo-se média de 0,090 e 9,09 $\mathrm{kg}$, respectivamente. Com a diminuição do CMS, esperava-se que os animais apresentassem um menor desempenho, em função do menor nível de ingestão de nutrientes, porém, este fato não ocorreu, provavelmente, porque as quantidades dos nutrientes das dietas, principalmente energia, possivelmente, foram suficientes para suprir as exigências de mantença dos animais, não influenciando $(\mathrm{P}>0,05)$ o desempenho $\mathrm{e}$, consequentemente, a conversão alimentar.

Na Tabela 7 são apresentados os resultados da análise econômica em relação ao ganho de peso diário, a fim de se verificar a viabilidade do uso da glicerina bruta na dieta, assim como, os custos fixos, operacionais e totais, relativos à produção de ovinos terminados em confinamento.

O valor do custo operacional efetivo, que descreve o quanto de recurso está sendo direcionado para cobertura de despesas, reduziu em função dos níveis de glicerina na dieta, este fato ocorreu em função do custo dos concentrados reduzirem, indicando que a glicerina bruta foi eficiente para minimizar custos. Outro fator relevante está relacionado ao menor consumo de matéria seca (CMS) dos animais que receberam dietas com níveis crescentes de glicerina. A diminuição do custo do concentrado que continha glicerina bruta, somado ao fato da diminuição do CMS pelos animais foi o fator determinante para diminuição do custo operacional efetivo.

O custo operacional total, que engloba a depreciação de benfeitorias, máquinas e equipamentos, apresentou o mesmo comportamento do custo operacional efetivo, sendo justificado pela mesma infraestrutura utilizada para todos os tratamentos. 


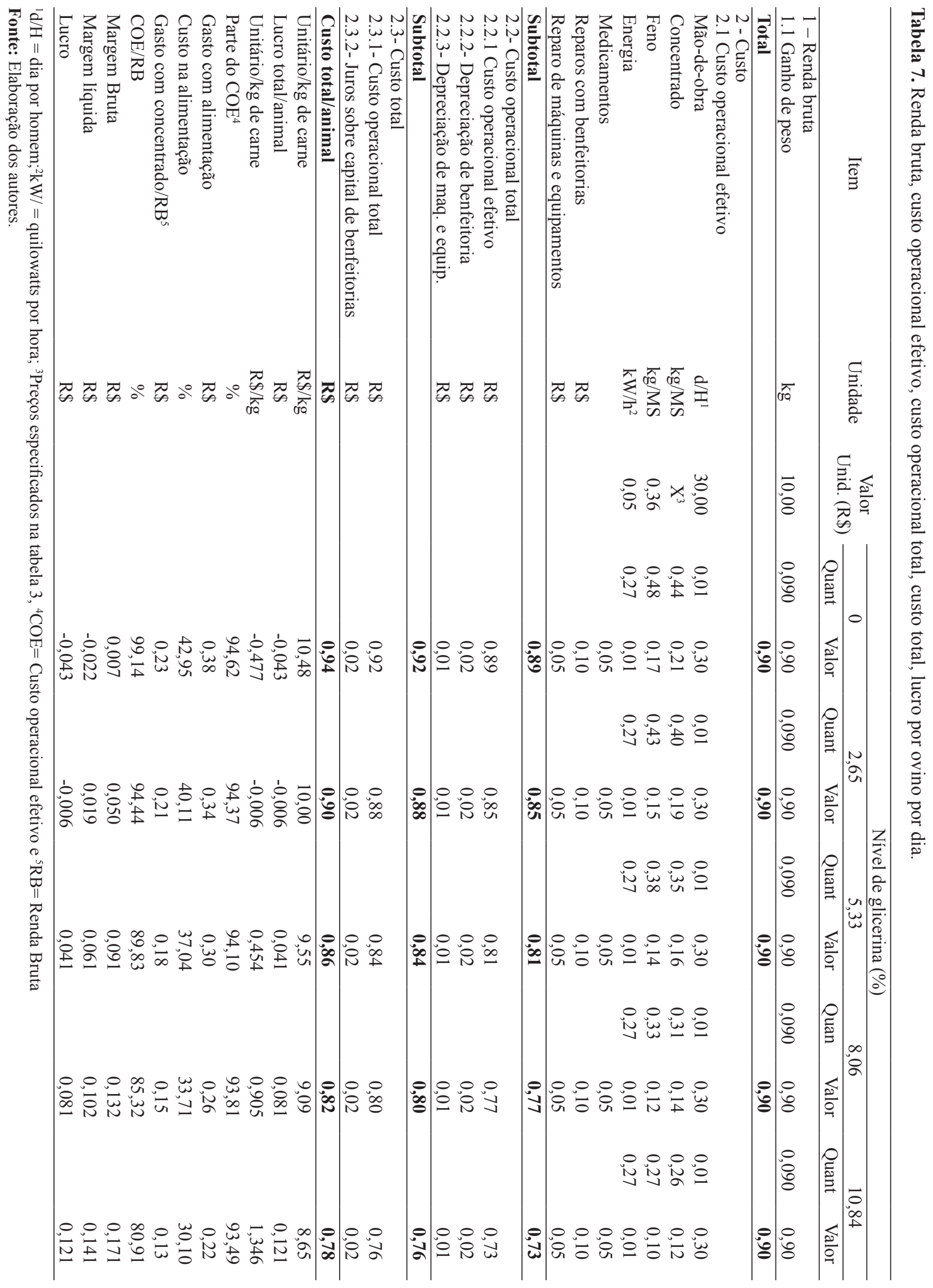


Musselman et al. (2008), avaliando os efeitos de inclusão da glicerina bruta $(0 ; 15 ; 30$ e $45 \%)$ na dieta de ovinos em terminação, sobre o desempenho e viabilidade econômica, afirmaram que cordeiros alimentados com o nível zero e $15 \%$ de GB na dieta tiveram uma ingestão de MS maior, comparado aos demais níveis, consequentemente, atingiram o peso de abate em média, com 23 dias a menos que os animais que receberam $30 \%$ de GB na dieta e 63 dias mais cedo do que os cordeiros sobre tratamento com $45 \%$ de GB. Os autores concluíram que o aumento do custo da alimentação, em função do aumento do número de dias que os animais com níveis maiores de $15 \%$ de GB na dieta precisaram para chegar ao peso de abate, representou inviabilidade econômica para ambos os tratamentos (30 e 45\%).

A margem bruta é obtida através da subtração da renda bruta pelo custo operacional efetivo. Foi observado que os valores da margem bruta por animal elevaram-se com os níveis crescentes de glicerina na dieta. Este fato ocorreu em função do custo operacional efetivo apresentar comportamento inverso, proporcionando uma margem de lucro maior para os tratamentos que utilizaram a glicerina na dieta.

A lucratividade por animal aumentou com a inclusão da glicerina na dieta, porém, os animais, de uma forma geral, não apresentaram desempenho satisfatório, o que acarretou em menor produto cárneo disponível para comercialização, este fato, aliado ao valor praticado no mercado de Itapetinga - BA, durante o período em que o experimento foi realizado, refletiu no decréscimo da TIR (Tabela 8 ), sendo estes valores pouco atrativos para os produtores.

O cálculo da VPL demonstrou que este investimento não foi interessante para nenhum dos tratamentos, sendo inviáveis economicamente.

Tabela 8. Taxa interna de retorno mensal e valor presente líquido para taxas de retorno de 6; 10 e $12 \%$, respectivamente, para um ano.

\begin{tabular}{lccccc}
\hline & \multicolumn{5}{c}{ Níveis de glicerina (\%) } \\
\cline { 2 - 6 } & 0 & 2,65 & 5,33 & 8,06 & 10,84 \\
\hline TIR (\%) & 0,49 & 0,36 & 0,23 & 0,10 & $-0,02$ \\
VPL 6\% & $-18,49$ & $-212,24$ & $-399,99$ & $-518,74$ & $-757,48$ \\
VPL 10\% & $-490,42$ & $-678,60$ & $-860,94$ & $-1037,45$ & $-1208,11$ \\
VPL 12\% & $-724,22$ & $-909,60$ & $-1089,22$ & $-1263,10$ & $-1431,22$ \\
\hline
\end{tabular}

Fonte: Elaboração dos autores.

\section{Conclusão}

No presente estudo, a utilização dos níveis de inclusão de glicerina bruta na dieta de cordeiros na fase de terminação em confinamento não apresentou viabilidade econômica para todos os tratamentos.

\section{Agradecimentos}

Ao meu orientador Jair de Araújo Marques (in memorian), a Universidade Estadual do Sudoeste da
Bahia - UESB e ao Programa de Pós-Graduação em Zootecnia da UESB - Campus Itapetinga.

\section{Referências}

CONTADOR, C. R. Indicadores para seleção de projetos. In: CONTADOR, C. (Ed.). Avaliação social de projetos. 2. ed. São Paulo: Atlas, 1988. p. 41-58.

GUNN, P. J.; SCHULTZ, A. F.; VAN EMON, M. L; NEARY, M. K; LEMENAGER, R. P.; PAS, C. P. Effects of elevated crude glycerin concentrations on feedlot performance, carcass characteristics, and serum 
metabolite and hormone concentrations in finishing ewe and wether lambs. The Professional Animal Scientist, Champaign, v. 26, n. 3, p. 298-306, 2010.

KERR, B. J.; HONEYMAN, M.; LAMMERS, P.; HOYER, S. Feeding bioenergy coproducts to swine: crude glycerol. Ames: Iowa State University, 2007. 11 p. Disponível em: <http://www.ipic.iastate.edu/ publications/IPIC11b.pdf 2007>. Acesso em: 2 abr. 2012.

LAGE, J. F.; PAUlinO, P. V. R.; PEREIRA, L. G. R.; VALADARES FILHO, S. C.; OLIVEIRA, A S.; DETMANN, E.; SOUZA, N. K. P.; LIMA, J. C. M. Glicerina bruta na dieta de cordeiros terminados em confinamento. Pesquisa Agropecuária Brasileira, Brasília, v. 45, n. 9, p. 1012-1020. 2010.

MATSUNAGA, M.; BEMELMANS, P. F.; TOLEDO, P. E. N.; DULLEY, R. D.; OKAWA, H.; PEDROSO, I. A. Metodologia de custo de produção utilizado pelo IEA. Agricultura em São Paulo, São Paulo, v. 23, n. 1, p. 123139, 1976.

MUSSELMAN, A. F.; VAN EMON, M. L.; GUNN, P. J.; RUSK, C. P.; NEARY, M. K.; LEMENAGER, R. P.; LAKE, S. L. Effects of crude glycerin on feedlot performance and carcass characteristics of market lambs. In: WESTERN SECTION, AMERICAN SOCIETY OF ANIMAL SCIENCES, 2008, West Lafayette. Proceedings... West Lafayette: Purdue University Department of Youth Development and Agricultural Education and Department of Animal Sciences, 2008. p. 353-355.
NATIONAL RESEARCH COUNCIL - NRC. Nutrient requirements of small ruminants. Washington: National Academy Press, 2007. 362 p.

NORONHA, J. F. Projetos agropecuários: administração financeira, orçamentos e viabilidade econômica. 2. ed. São Paulo: Atlas, 1987. v. 1, 269 p.

PARSONS, G. L.; SHELOR, M. K.; DROUILLARD, J. S. Performance and carcass traits of finishing heifers fed crude glycerin. Journal of Animal Science, Champaign, v. 87, n. 2, p. 653-657, 2009.

PERES, A. A. C.; SOUZA, P. M.; MALDONADO, H. SILVA, J. F. C.; SOARES, C. S.; BARROS, S. C. W.; HADDADE, I. R. Análise econômica de sistemas a pasto para bovinos no Município de Campos dos GoytacazesRJ. Revista Brasileira de Zootecnia, Viçosa, MG, v. 33, n. 6, p. 1557-1563, 2004.

PYATT, N. A.; DOANE, P. H.; CECAVA, M. J. Effect of crude glycerin in finishing cattle diets. Journal of Animal Science, Champaign, v. 85, n. 2, p. 412, 2007. Supplement 1.

SAEG. Sistema para análises estatísticas. versão 9.1 . Viçosa: UFV, 2007. 
